\title{
Resultados de la corrección de la Clase l| con un bionator modificado
}

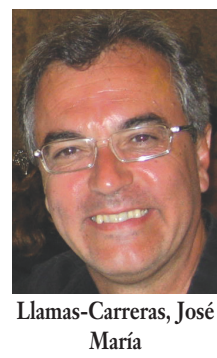

Results of Class I| correction with a modified Bionator appliance

\section{LLamas-Carreras, José María* Bravo-González, Luis Alberto**}

*Doctor en Medicina y Cirugía. Especialista en Estomatología. Práctica exclusiva ortodoncia en Sevilla.

**Profesor Titular. Unidad Docente de Ortodoncia. Clínica Odontológica Universitaria. Universidad de Murcia.

\section{Correspondencia}

José María Llamas

Ramón de Carranza 32,

41011 - Sevilla
Resumen. Se han analizado pacientes que fueron corregidos de Clase II con retrognatia mandibular. Era condición de inclusión en el estudio que los individuos de la muestra presentaran normoclusión dentaria después de más de cuatro años de finalizado el tratamiento ortodóncico. Se analizaron los efectos del bionator en determinadas variables cefalométricas, obteniendo resultados que reflejan ligeros cambios favorables en las posiciones sagitales de los maxilares y cambios evidentes a nivel de las inclinaciones de los incisivos superiores e inferiores que facilitan la corrección de la Clase II.

Palabras clave. Maloclusión de Clase II, Cambios ortopédicos, Bionator.

Abstract. We analyzed patients with retruded mandible and class II malocclusion, treated with a Bionator appliance in a first orthodontic phase. The patients were included in the study if they had stable normocclusion more than four years after completion of the whole treatment. Results show favourable, but light, changes in sagittal relations of both jaws, and significant changes in the proclination of both incisors, that helped to correct class II malocclusion.

Key words. Class II malocclusion, Orthopedic changes, Bionator appliance.

\begin{tabular}{ccc}
\hline Fecha recepción & Fecha última revisión & Fecha aceptación \\
$25-4-2000$ & $26-2-2001$ & $19-3-2001$ \\
\hline
\end{tabular}

BIBLID [1138-123X (2005)10:1; enero-febrero 1-124]

Llamas Carreras JM, Bravo-González La. Resultados de la corrección de la Clasell con un bionator modificado. RCOE 2005;10(1):21-28. 


\section{Introducción}

La maloclusión de Clase II en adolescentes presenta una prevalencia del $15 \%$, siendo la segunda alteración dentooclusal más encontrada después del apiñamiento dentario'. Esta prevalencia hace referencia a la población general, ya que en población que demanda tratamiento ortodóncico la situación es otra, siendo las cifras incluso superiores ${ }^{2,3}$. En nuestro país, un estudio acerca de la epidemiología de las maloclusiones en gran parte del territorio nacional, arroja la cifra del $51 \%$ de pacientes con relación de Clase II división de $1^{\text {a }}$ entre los que acuden a las consultas de ortodoncia ${ }^{4}$. Hoy día es común aceptar que la Clase II es una denominación clasificatoria en la que se definen diversas entidades dentofaciales diferentes que solo tienen en común una relación distal de la arcada inferior con respecto a la superior5. Por ello el tratamiento de la Clase II con resalte incisivo aumentado presenta distinta aproximación en base a la identificación morfotipológica esquelética y dentaria de esta maloclusión. Sin embargo, los autores consultados están de acuerdo en que la mayor parte de las Clases II se deben al déficit de crecimiento sagital de la mandíbula ${ }^{3-10}$.

El tratamiento de la maloclusión de Clase II división 1 a presenta así, durante la fase de crecimiento, una serie de alternativas en base a las relaciones sagitales y verticales de los maxilares con respecto a la base craneal. Nuestra elección terapéutica variará frente a unas características faciales de crecimiento vertical u horizontal. Será distinto el tratamiento en el caso de que nos encontremos frente a una retrog- natia mandibular o a una protrusión del maxilar superior, o incluso frente a una correcta relación intermaxilar. Pero es incluso más importante la valoración de la relación vertical de ambos maxilares, que define la tendencia y el patrón de crecimiento en altura facial. Si la Clase II que deseamos tratar se presenta en una cara de crecimiento horizontal, observaremos generalmente que la relación entre las alturas faciales estará desequilibrada, con una ratio que denota un mayor crecimiento posterior que anterior. La situación opuesta es la que encontraremos cuando exista un crecimiento vertical. En ambos casos, el tipo de crecimiento vertical se convierte en la cualidad facial que imprime carácter a la maloclusión. Así, reconocemos con Schudy ${ }^{11^{*}}$ que es importante que la profesión esté de acuerdo acerca del criterio más importante a la hora de seleccionar los tipos faciales... porque los tipos de morfología facial se identifican con tipos específicos de maloclusión, en una especie de síndrome dentofacial...

En un número importante de pacientes la corrección de la Clase II pasa por el avance mandibular puro, intentando respetar la altura facial inferior, tanto la anterior como la posterior. Para ello la aproximación terapéutica va de la mano de la utilización de determinados aparatos ortopédicos, uno de los cuales es el bionator. En el año 1952, Balters construyó el bionator no sólo para conseguir un efecto de avance mandibular sino también para asegurar y mejorar el cierre labial y el habitáculo lingual. Tal y como lo utilizamos en la clínica habitual y en este estudio que presentamos el bionator modificado solo busca crear una mordida protrusiva en la que los incisivos superiores y los inferiores queden sujetos por el arco labial superior y por la resina acrílica inferior. Su uso queda confinado a las etapas de pico de crecimiento puberal, como fase inicial del tratamiento ortodóncico con aparatos fijos, y su efecto se pretende que consista en potenciar el crecimiento mandibular, sin afectar al crecimiento sagital del maxilar superior $^{12^{*}}$.

\section{Evidencias científicas 丩 planteamiento del rrabajo}

Iniciamos esta revisión y actualización de los efectos del bionator citando el trabajo, ya clásico, de Aelbers y Dermaut ${ }^{13^{* *}}$, en el que establecieron que sólo el aparato de Herbst, tal y como demuestran los trabajos de Pancherz ${ }^{14 *}$, arrojaron resultados proclives al demostrar aumentos significativos, tanto clínicamente como estadísticamente, de crecimiento mandibular más allá de lo esperado. Coincidimos con Kragt y Duterloo ${ }^{15}$ en que ortopedia es todo cambio en la posición, cantidad y/o dirección, de los huesos en relación con el esqueleto, permanente en el tiempo. Es en la década de los años sesenta del siglo pasado, cuando los trabajos de Harvold ${ }^{16 * *}$ con el activador despertaron el interés de los ortodoncistas estadounidenses acerca de las posibilidades de influir de manera favorable en el tamaño y posición de la mandíbula. Sin embargo, bien pronto, en 1979, un sólido trabajo de Wieslander y Lagerstgrom ${ }^{17 * *}$ concluyó que con el uso del activador no se producía incremento de la longitud mandibular 


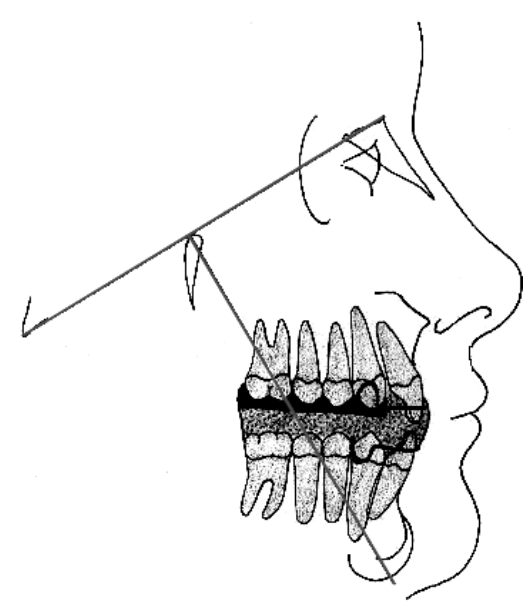

Figura 1. Posición protrusiva del bionator modificado respetando el eje facial de crecimiento.

más que temporalmente, y que el incremento, en todo caso, era sólo de $1,5 \mathrm{~mm}$, lo que no presentaba significado clínico alguno. En 1988, un trabajo nuestro llega a conclusiones similares, determinando que tras la utilización del activador en una muestra de pacientes en Clase II con cara corta, la rotación mandibular que se produjo fue favorable, para posteriormente recidivar hasta posiciones similares a las inicialmente medidas18. Es por ello que decidimos alterar la construcción del activador disminuyendo la altura del bloque posterior de acrílico de manera que se mantuviera cerca de la dimensión vertical de reposo, tomada con los incisivos en contacto (fig. 1). De esta manera buscábamos evitar el efecto de rotación mandibular para incidir terapéuticamente en los cambios sagitales de la dentición maxilar inferior. Esta construcción se acerca a la altura que define al bionator, razón por la cual consideramos que el aparato ortopédico que utilizamos es un bionator modificado (figs. $2 a$ y $2 b$ ).
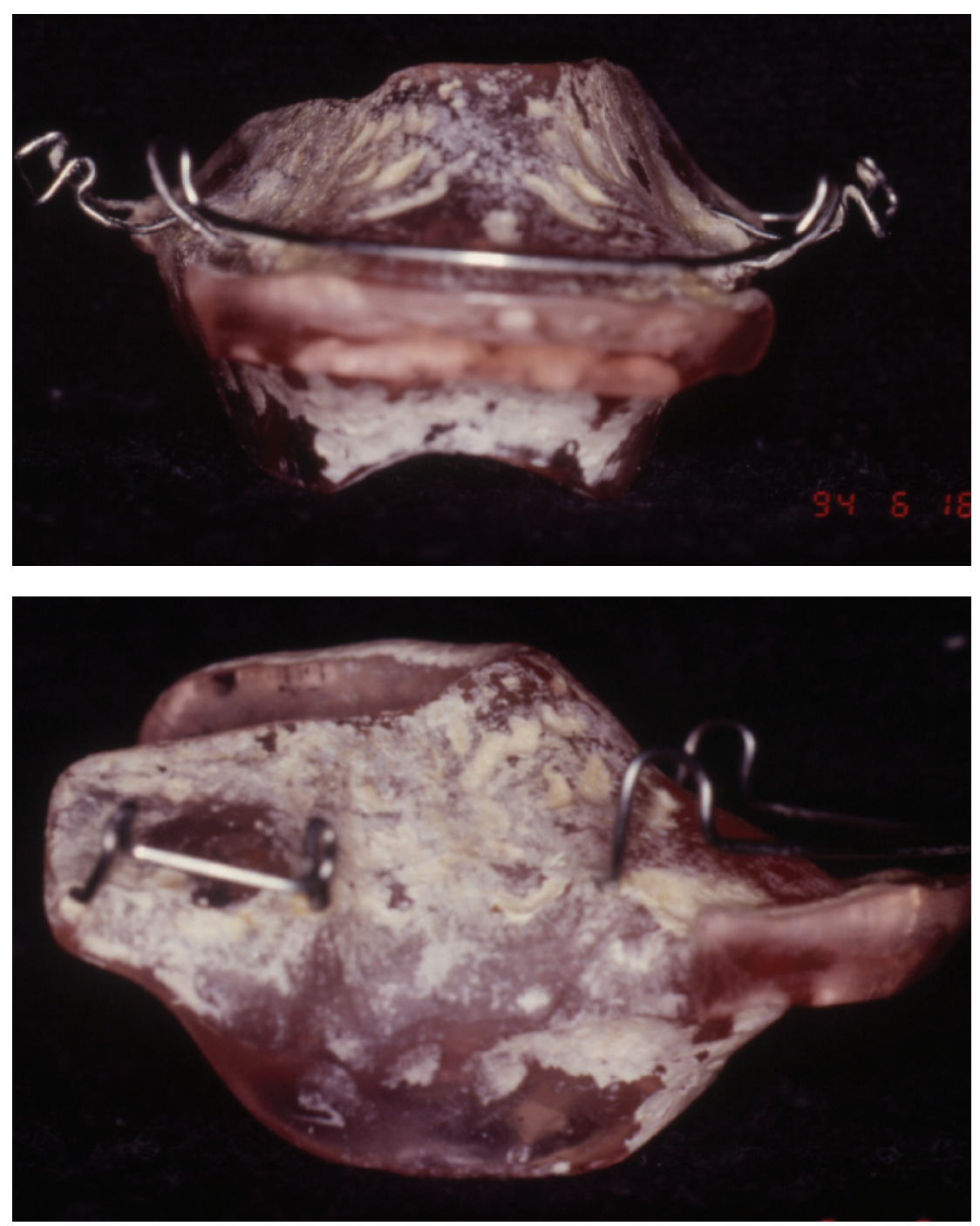

Figura 2 (a y b). Bionator modificado de avance mandibular.

Gunner y cols ${ }^{19^{* \star}}$ constataron que después de seis meses de tratamiento con un bionator combinado con aparatos fijos, la protrusión mandibular aumentó y la actividad ósea en el espacio articular se incrementó, indicando una respuesta adaptativa al uso de la terapéutica. A esto oponen los trabajos anteriormente citados que estos resultados a corto plazo sobre la actividad ósea no significan una man- díbula finalmente mayor ${ }^{13^{* *}, 17^{* \star}}$. Una de las cuestiones más debatidas es si el uso del bionator en una posición protruida produce traslación del cóndilo fuera de la fosa con alteraciones discales indeseables. Sin embargo, Watted y cols, en 2001, en una muestra de pacientes adolescentes que usaron el bionator durante 12 meses constataron, por medio de resonancia magnética, que la relación entre el disco y 


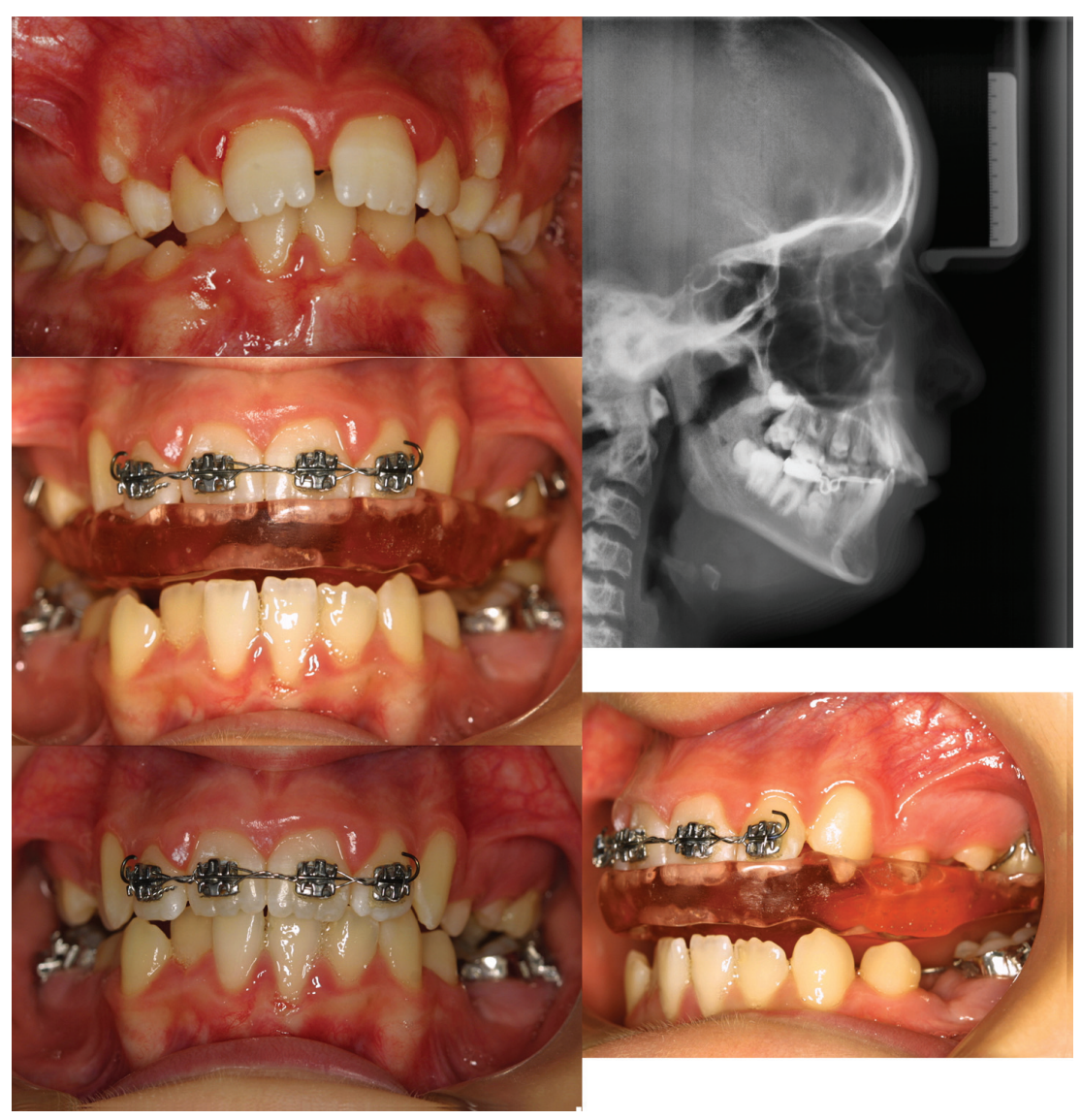

Figura 3. Bionator usado como fase inicial junto a aparatos fijos superiores.

el cóndilo se mantuvo normal ${ }^{20^{* *}}$, y que la posición del cóndilo con respecto a la fosa no fue más adelantada, sino ligeramente más distal. Esto coincide con resultados obtenidos anteriormente por nosotros en un trabajo acerca de resultados terapéuticos con activador $^{21}$

El uso del bionator recomendado a los pacientes de este trabajo fue de un mínimo de doce horas diarias, aconsejando que al menos cuatro horas fueran de uso no nocturno. En la actualidad sabemos que el máximo efecto del bionator se consigue con el uso no nocturno $0^{22^{*}}$ ya que es entonces cuando se consigue un mayor efecto sobre los músculos maseteros y suprahioideos. Sigue siendo controvertido si más que crecimiento de las bases óseas inferiores existe un efecto de mesialización de toda la dentición inferior, lo que justificaría que se demuestre mayor frecuencia de erupción de terceros molares inferiores tras el uso del bionator ${ }^{23^{*}}$.

Esto fue lo que nos llevó a desear comprobar los efectos del bionator en las relaciones esqueléticas y dentarias sobre los pacientes de Clase II con retrognatia mandibular. Utilizamos este aparato para evitar inducir una rotación mandibular antihoraria ${ }^{24 *}$ más propia del uso del activador.

\section{Material 4 mérodo}

El bionator se utilizó en la muestra que a continuación indicaremos en la fase inicial del tratamiento ortodóncico de manera previa o coincidente con los primeros aparatos fijos (fig. 3). Para analizar los efectos dentoesqueléticos del bionator seleccionamos 30 pacientes que habiendo utilizado dicho aparato como fase inicial del tratamiento ortodóncico, presentaran Clase I molar y canina, resalte y sobremordida normal, y mantenimiento de correctas líneas faciales del perfil más de cuatro años después de finalizado el mismo. El tiempo medio de utilización del bionator fue de aproximadamente 12 meses. Nuestro objetivo fue conocer las modificaciones que en estos individuos habían permitido la corrección de la Clase II con retrognatismo del maxilar inferior. Entre los pacientes estudiados encontramos casos tanto de sobremordida como de mordida abierta, como podemos observar en las figuras 4 y 5 .

Los registros utilizados para este estudio fueron las telerradiografías tomadas todas con el mismo aparato y con la misma magnificación, descartándose aquellos pacientes que no presentaran registros adecuados en alguno de los estadíos del tratamiento. Se realizaron los trazados cefalométricos y se midieron las siguientes variables (fig. 6):

Variables de relaciones sagitales maxilares: SNA, SNB y ANB.

Variables dentomaxilares: inclinación del incisivo inferior y del incisivo superior.

Variables de relaciones verticales: ángulo gonial, plano mandibular, relación maxilo-mandibular. 


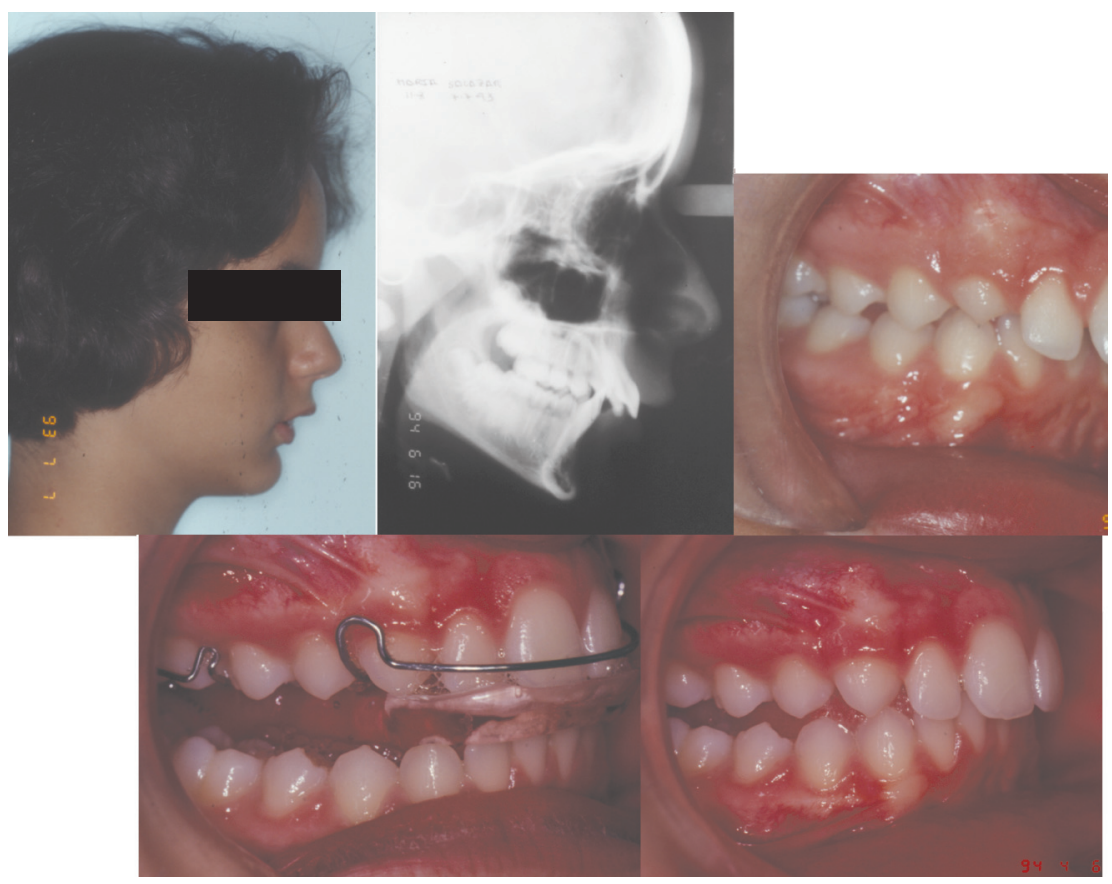

Figura 4 a. Fase inicial con bionator y avance mandibular.

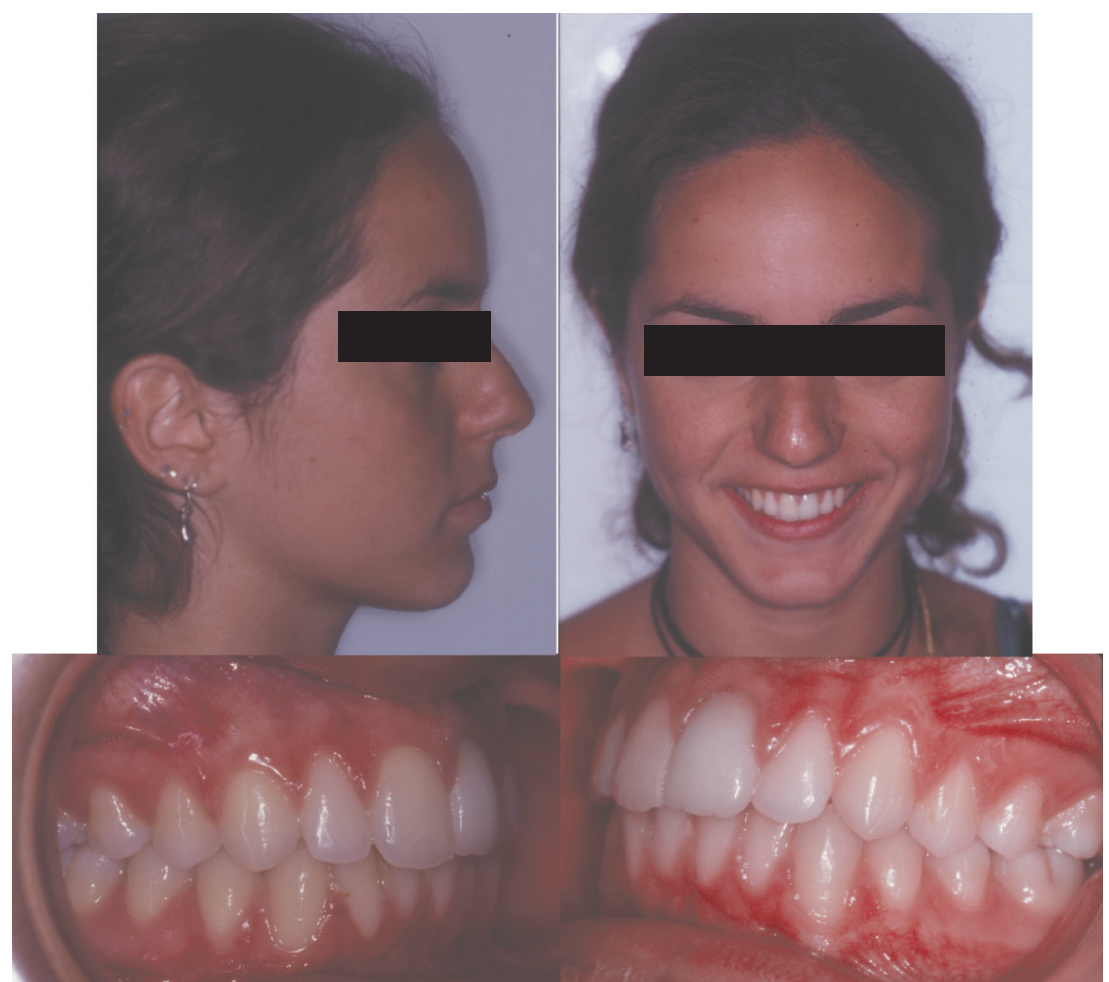

Figura 4 b. Paciente anterior cuatro años después de finalizar el tratamiento ortodóncico.

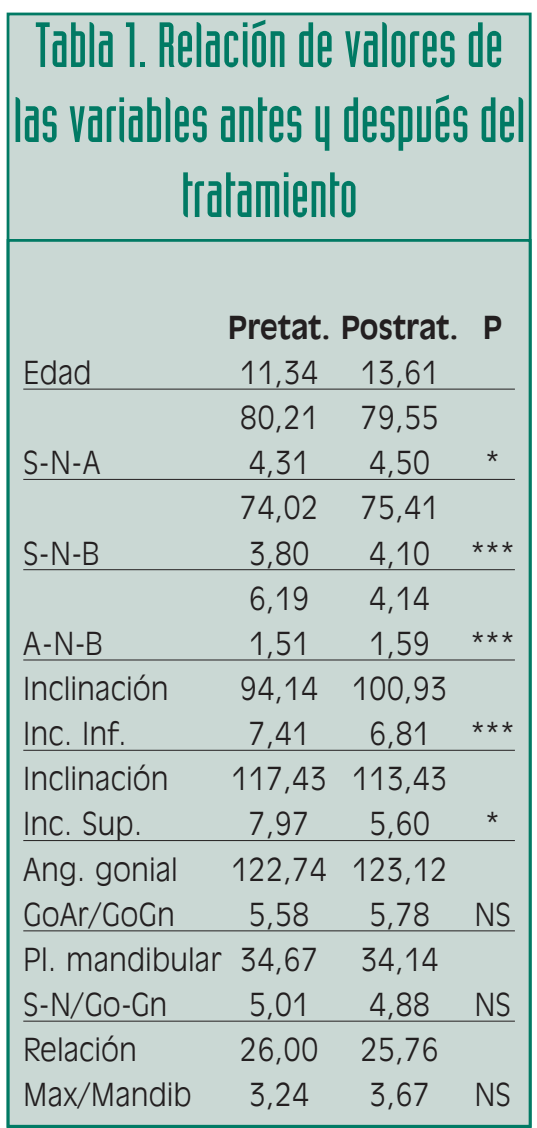

\section{Resullados u discusión}

Observamos en la tabla 1 los resultados de los valores de las variables en los estadíos previos y posteriores al tratamiento ortodóncico. Asimismo expresamos las diferencias significativas entre los valores en ambas observaciones a través del valor estadístico p. Encontramos una mejor relación sagital intermaxilar (ángulo ANB) a expensas de cambios ligeros, aunque estadísticamente significativos, de los ángulos SNA y SNB. Concretamente el ángulo SNA disminuyó menos de un grado, mientras que el ángulo SNB aumentó casi un grado y medio. El ángulo ANB, que era de 6,2 grados, acabó siendo de poco más de cuatro 


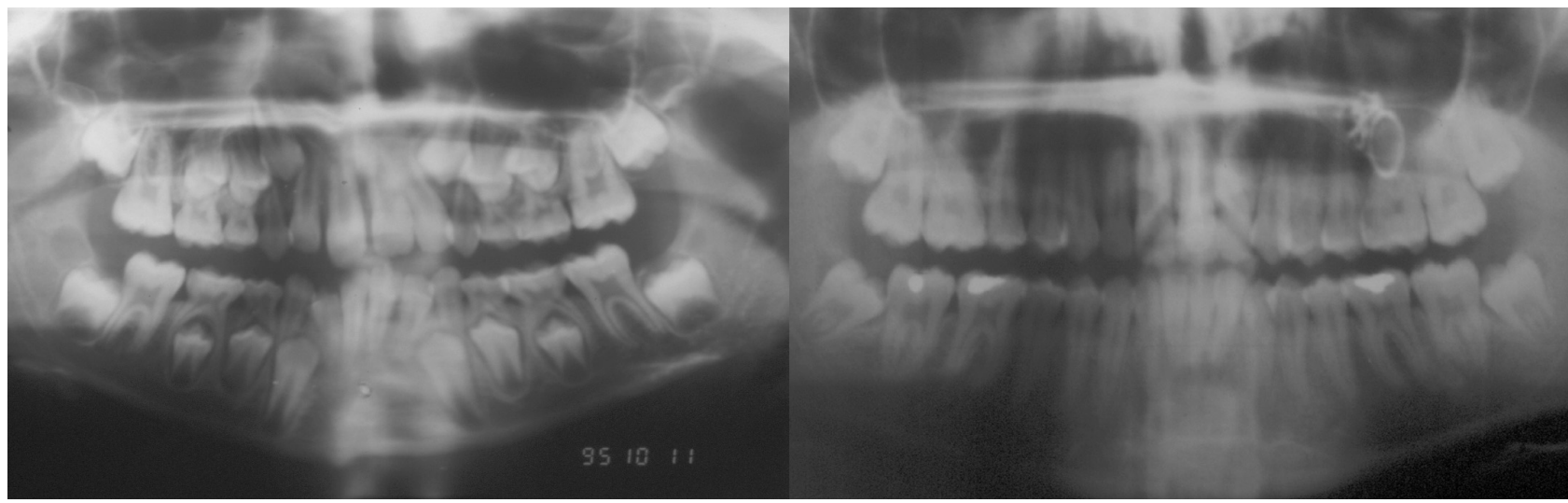

Figura 4 c. Comparación de radiografías panorámicas de la paciente anterior, a los 10 y a los 17 años, observándose el aumento del espacio de erupción de los terceros molares inferiores.

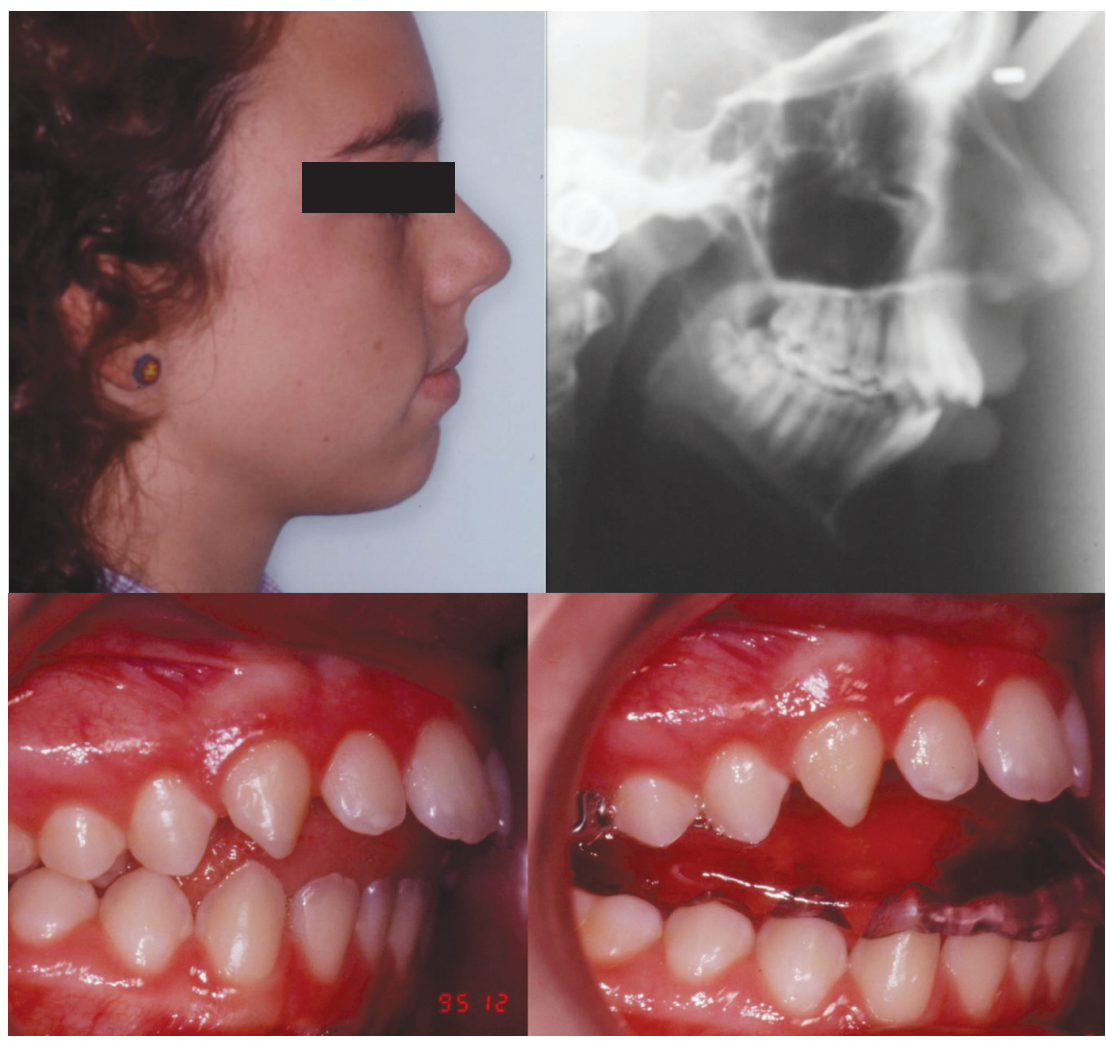

Figura 5 a. Paciente con Clase II y mordida abierta antes del tratamiento y con el bionator inicial.

grados. Podríamos pues discutir la aseveración de autores anteriormente citados ${ }^{13^{* *}, 1^{7 * *}}$ de que no son cambios que supongan efectos clínicos apreciables. Recordemos que la muestra consiste en individuos de Clase II cuya distoclusión inferior quedaba no solo corregida sino que era estable en el tiempo. Con ello pretendíamos ajustarnos a la definición antes citada de ortopedia como cambios en la posición, cantidad o dirección mandibular permanente en el tiempo.

Si bien los cambios en las posiciones sagitales de los maxilares existieron, aunque no fueron notables, los cambios verticales del tercio inferior de la cara no se manifestaron ni expresaron significación estadística alguna. Las alturas faciales inferiores permanecieron inalterables en lo que respecta al ángulo gonial mandibular, plano mandibular y relación angular maxilomandibular, medida entre el plano mandibular y el plano maxilar. Esta era nuestra intención al elegir el efecto ortopédico del bionator que prima el avance mandibular sobre los cambios en la dimensión vertical facial inferior.

Las estructuras anatómicas que más cambios angulares mostraron tras el tratamiento de ortodoncia son los incisivos, tanto superiores como inferiores. De hecho, el incisivo inferior se volcó más de seis grados mientras que el incisivo superior se retroinclina cuatro grados. Todo ello resulta en un cambio del ángulo interincisivo de más de 11 grados, lo que claramente debe contribuir a la corrección de la distoclusión. 

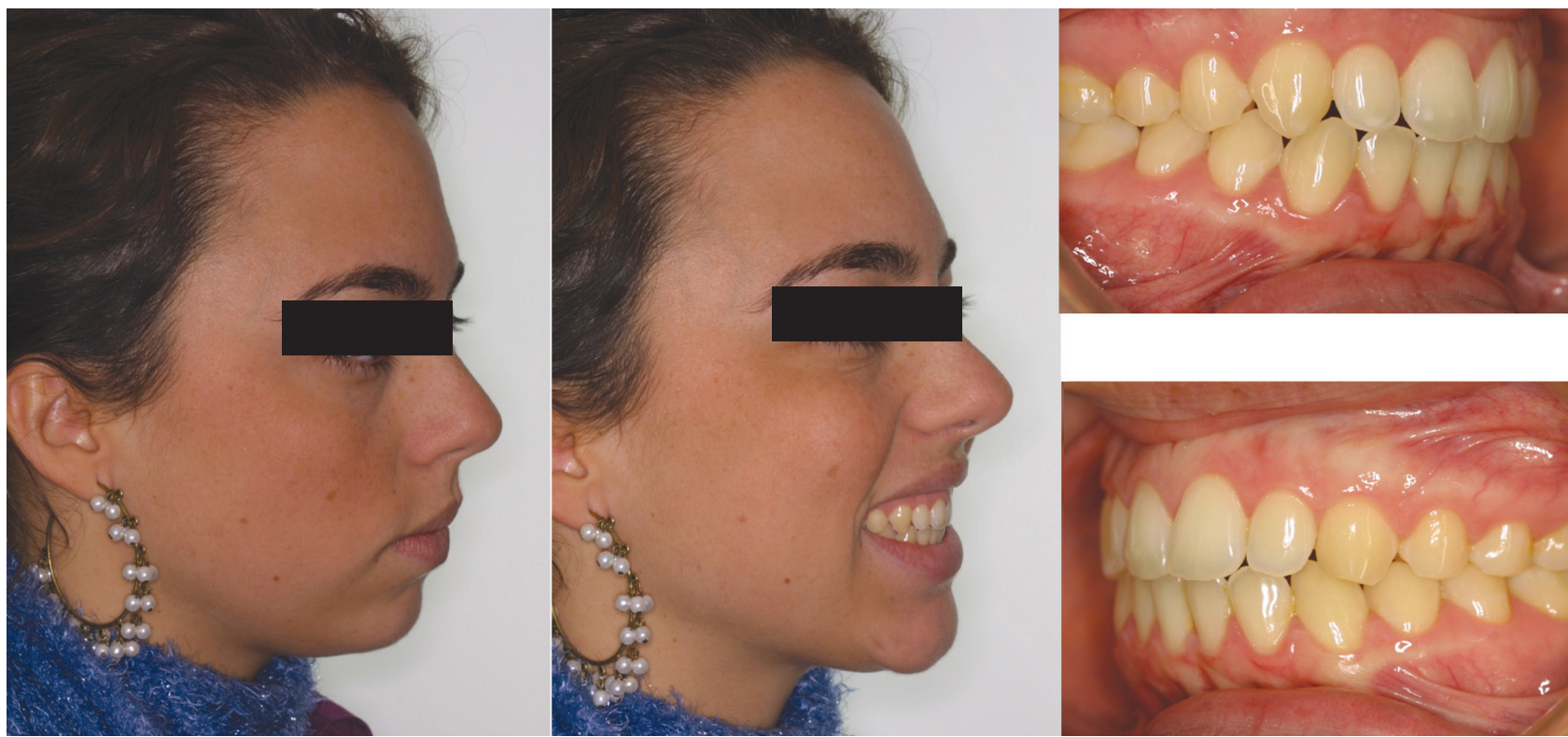

Figura 5 b. Paciente anterior más de cinco años tras la finalización del tratamiento.

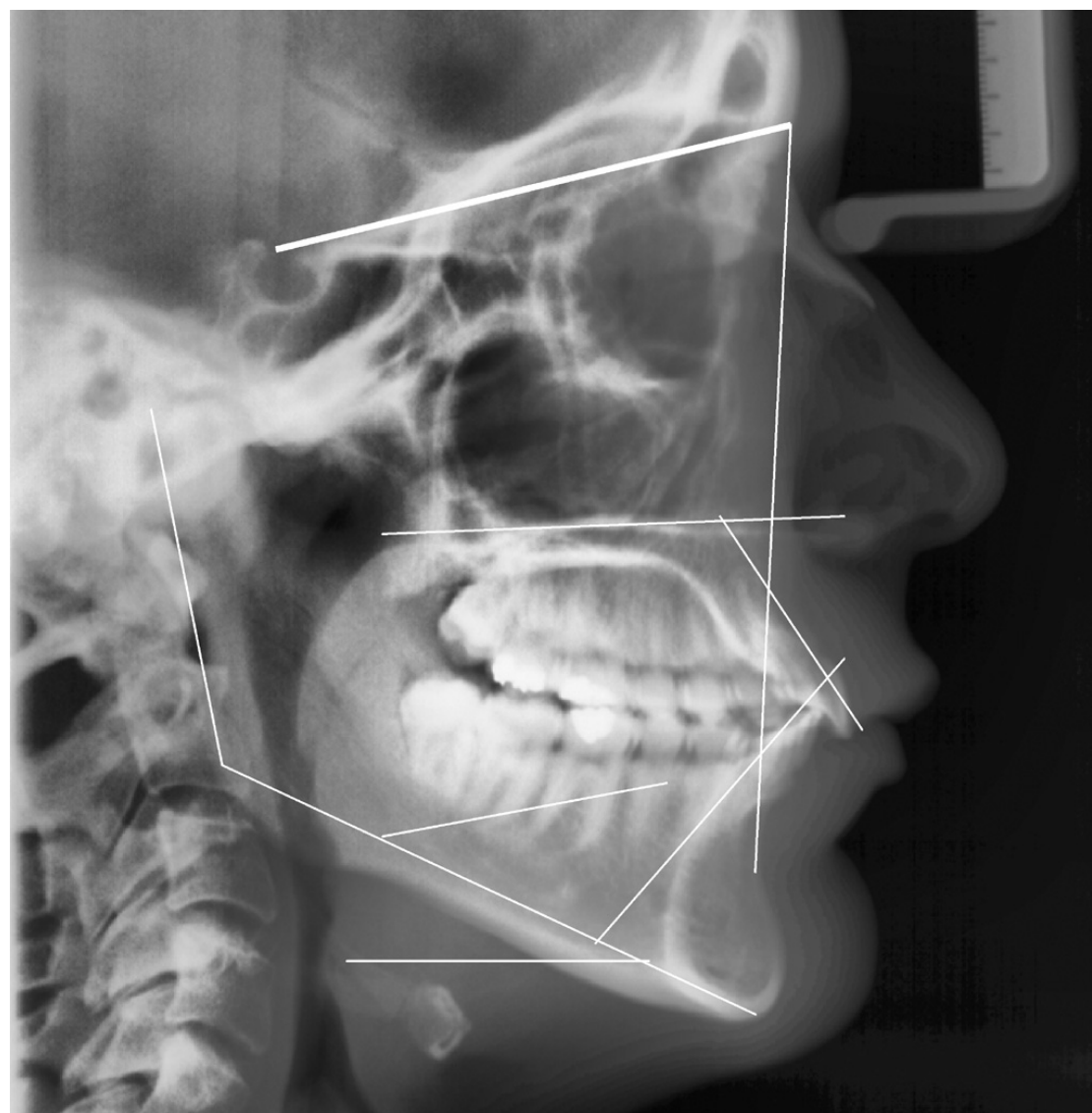

Figura 6. Planos utilizados para estudiar las variables de este estudio.
Podemos colegir, por lo tanto, de los resultados que la corrección de la Clase II con retrognatia mandibular existente en los pacientes de la muestra estudiada se ha debido producir por un conjunto de cambios sagitales de los maxilares y, sobre todo, de inclinaciones de los incisivos a favor de la eliminación del resalte. Todo ello indica cambios dentoalveolares que en la muestra analizada han sido estables en el tiempo. En un estudio posterior registraremos las variables en la situación de estabilidad para analizar si este conjunto de cambios dentoalveolares favorables se mantienen en el tiempo y si, por ello, son los responsables de la estabilidad encontrada.

\section{Conclusiones}

1. La muestra estudiada presenta ligeros cambios sagitales tras el uso del bionator en las posiciones de los maxilares que van en el sentido de la correc- 
ción de la Clase II, siendo mayor el avance mandibular que la retrusión maxilar.

2. Los mayores efectos de compensación de la Clase II se observan, tras el uso del bionator, en las inclinaciones de los incisivos, con proinclinación del inferior y retroinclinación del superior. 3. Los efectos terapéuticos del bio- nator en nuestra muestra indican compensaciones dentoalveolares que contribuyen a la corrección de la maloclusión de clase II.

\section{Bibliografila recomendada}

Para profundizar en la lectura de este tema, el/los autor/es considera/an interesantes los artículos que aparecen señalados del siguiente modo: *de interés ${ }^{*}$ de especial interés.

1. Kelly J, Harvey C. An assesment of the teeth of youths 12-17 years. En DHEW Pub No. (HRA). Washington DC. National Center for Health Statistics, 1977:77-1644..

2. Proffit WR, Phillips C, Dann C. Who seeks surgical-orthodontic treatment? The characteristics of patients evaluated in the UNC Dentofacial Clinic. Int J Adult Orthod Orthognath Surg 1990;5:153-60.

3. Mc Namara JA. Components of class II malocclusion in children 8-10 years of age. Angle Orthod. 1981;51:177-202.

4. Bravo LA. Análisis del patrón facial y de su relación con la maloclusión. Tesis Doctoral. Universidad de Valencia, Facultad de Medicina. Valencia, 1986.

5. Canut JA. Ortodoncia clínica. Barcelona:Salvat Eds, 1988

6. Costa A. Análisis de Steiner en la raza espanola. Rev Esp Ortod 1972;4:205-22.

7. Cox H, Van der Linden, F. Facial harmony. Am J Orthod 1971;59:175-83.

8. Llamas JM, Asensi, C. 1986. Análisis cefalométrico del perfil blando facial en individuos maloclusivos. Rev Esp Ortod 1986;16:57-68.

9. Llamas JM. EI perfil blando en las maloclusiones. Un estudio cefalométrico comparativo. Rev Esp Ortod 1988;18:135-46.

10. Martínez Miñana P. Análisis cefalométrico de las oclusiones ideales en la población valenciana. Tesis de Licenciatura. Universidad de Valencia, Facultad de Medicina. Valencia, 1981

11*. Schudy FF. The rotation of the mandible resulting from growth. Its implication in orthodontic treament. Angle Orthod 1965;35:36-50.

Es un trabajo clásico, importante para la comprensión de la importancia del patrón facial vertical en las maloclusiones.

12*. Carels C, Reychler A, Van der Linden F. Cephalometric evaluation of dento-skeletal changes during treatment with the Bionator Type 1. J Oral Rehab 1997;24:841.

En este trabajo bien desarrollado se analizan, sin encontrarlas, diferencias entre efectos esqueléticos del bionator en pacientes con mordidas abiertas y profundas.

$13^{* *}$. Aelbers CMF, Dermaut LR. Orthopedics in orthodontics: fiction or reality? A review of the literature. Part I. Am J Orthod 1996;110: 513-5.

En este trabajo ya clásico, a pesar de su reciente aparición, se configura un extenso y provocador meta-análisis acerca de los efectos de los aparatos ortopédicos.

14*. Pancherz H. Dentofacial Orthopedics or Orthognathic Surgery: Is It a Matter of Age? Am J Orthod Dentofac Orthop 2000;117:571-4. Consideramos interesante su lectura para limitar las indicaciones de la cirugía de avance mandibular.

15. Kragt G, Duterloo HS. The Initial Effects of Orthopedic Forces: A Study of Alterations in the Craniofacial Complex of a Macerated Human Skull Owing to Highpull Headgear Traction. Am J Orthod 1982;81:57-64.

16**. Harvold EP, Vorgervik K. Morphogenetic response to activator treatment. Am J Orthod 1971;60:478-90.

Es un trabajo clásico con el que se inicia el interés de la ortodoncia norteamericana por los aparatos funcionales y ortopédicos.

$17 * *$. Wieslander L, Lagerstrom L. The effect of activator treatment on class II malocclusions. Am J Orthod 1979;75:20-6.

Son interesantes sus resultados y conclusiones, aunque signifiquen discrepancias entre los hallazgos de la investigación y de los resultados clínicos.

18. Llamas JM. Activador de Harvold y estabilidad. Un acercamiento a los mecanismos dentofaciales implicados. Rev Esp Ortod 1998;28:117-27.

19**. Guner DD, Ozturk Y, Sayman HB. Evaluation of the Effects of Functional Orthopaedic Treatment on Temporomandibular Joints With Single-photon Emission Computerized Tomography. Eur J Orthod 2003;25:9-12.

En este trabajo, de sofisticada y vanguardista metodología, encontramos claramente resuelta la mayor actividad metabólica con el uso de los aparatos funcionales. La duda, empero, permanece: ¿estos efectos producen, a largo plazo, en una mandíbula mayor?

20**. Watted N, Witt E, Kenn. The Temporomandibular Joint and the Disc-Condyle Relationship After Functional Orthopaedic Treatment: A Magnetic Resonance Imaging Study. Eur J Orthod 2001;23:683-93.

Aconsejamos la lectura de este trabajo para incrementar el conocimiento acerca de la adaptación de las estructuras de la ATM a los cambios posicionales de la mandíbula en crecimiento.

21. Bravo LA, Llamas J.M. Crecimiento mandibular y acción terapéutica: un análisis comparativo. Rev Esp Ortod 1984;14:131-8.

$22 *$. Hiyama S, Kuribayashi G. Nocturnal Masseter and Suprahyoid Muscle Activity Induced by Wearing a Bionator. Angle Orthod 2002;72:48-54.

Este trabajo abunda en la mayor eficacia de los aparatos de ortodoncia durante la vigilia.

23*. Dolce C, Fusco AD. Third Molar Position Following Bionator Treatment. Clin Orthod Res 2000;3:106-13.

Supone un original enfoque de inquietud investigadora, resuelto con seriedad.

$24 * *$. Bjork A. Prediction of mandibular growth rotation. Am J Orthod 1969;55:585-9.

Trabajo clásico en ortodoncia, del que ya está todo dicho. 